\title{
Novel Approach of Antithrombotic Potency Amongst Patients Admitted to Hospital with Bleeding Using HEMORR2HAGES Score: A Retrospective Cohort Study
}

\author{
Mohannad Alshibani, PharmD, BCPS ${ }^{1,2}$ \\ ${ }^{1}$ King Abdulaziz University, Faculty of Pharmacy, Department of Pharmacy Practice, Jeddah, Saudi Arabia; ${ }^{2}$ Center for Health Outcomes \\ and PharmacoEconomic Research, University of Arizona, Tucson, AZ, USA
}

\section{ABSTRACT}

Background: The purpose of this study was to evaluate whether a relationship exists between baseline HEMORR 2 HAGES score and antithrombotic potency amongst patients presenting with bleeding complication. We hypothesized that the more antithrombotic regimen potency, the less HEMORR_HAGES score you have.

Methods: This is a retrospective observational study of patients admitted with a diagnosis of active bleeding between November 1, 2013 and August 31, 2015. The antithrombotic groups included patients on the following regimens: single antiplatelet therapy (SAP), single oral anticoagulant therapy (SOAC), dual antiplatelet therapy (DAPT), dual combination (SOAC+SAP), and triple antithrombotic therapy. The primary outcome was to review the mean HEMORR $\mathrm{HAGES}_{2}$ score among the various groups.

Results: There were a total of 180 patients in the study. No significant difference was noted among the five groups

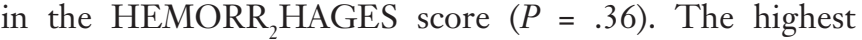
HEMORR $_{2}$ HAGES score was in the SAP group $(3.23 \pm 1.1)$. The lowest HEMORR,HAGES score was in the DAPT group $(2.59 \pm 1.2)$. In the Sub Group analysis, we compared single versus dual versus triple therapy, and we found the lowest HEMORR $\mathrm{HAGES}_{2}$ score in the triple therapy group $(2.70 \pm 1.6) ;(P=.29)$.

Conclusions: Among patients admitted with active bleed-

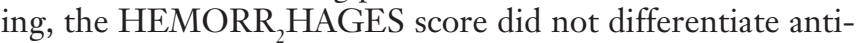
thrombotic potency amongst groups with various regimens. This study highlights the necessity to evaluate antithrombotic therapy according to benefits and harms.

\section{INTRODUCTION}

Background: There has been a marked increase in the number of antithrombotic therapies to treat patients with cardiovascular and thromboembolic events. With the increased use of several antithrombotic therapies, complications such as

Received Fune 30, 2019; accepted August 5, 2019.

Correspondence: Mobannad Alshibani, King Abdulaziz University, IBN Almagheera Street, Feddah, 23455, Saudi Arabia (e-mail: malshibani@kau.edu.sa). bleeding may result in significant morbidity [Lamberts 2012]. Current evidence supports the use of dual antiplatelet therapy after percutaneous coronary intervention (PCI) to reduce the risk of stent thrombosis or acute coronary syndrome (ACS), and the use of oral anticoagulants (OACs) to prevent cardioembolic neurologic events in patients with atrial fibrillation (AF) [D'Ascenzo 2013]. The use of OACs and dual-antiplatelet therapy (DAPT) is believed to increase the risk of fatal and nonfatal bleeding by retrospective analysis [Gage 2006]. The rate of bleeding is expected to be more frequent with the use of triple therapy (DAPT + OAC) from recent studies [Orford 2004; Andrade 2013; Hansen 2010; Sorensen 2009; Rubboli 2008]. To decrease the risk of bleeding with the use of antithrombotic therapy, the recent 2019 guideline for the management of atrial fibrillation considered the reduction of the duration of triple therapy to a period of 4-6 weeks

Table 1. Definition of HEMORR2HAGES score

\begin{tabular}{|c|c|}
\hline HEMORR2HAGES Risk Factors & Definition \\
\hline $\begin{array}{l}\text { Hepatic (1) or Renal Disease } \\
\text { (1) }\end{array}$ & $\begin{array}{c}\text { Cirrhosis; >two-fold AST or ALT; Crcl } \\
\qquad 30 \mathrm{~mL} / \mathrm{min}\end{array}$ \\
\hline Ethanol use (1) & $\begin{array}{c}\text { Alcohol abuse, recent hospitalization for } \\
\text { alcoholism }\end{array}$ \\
\hline Malignancy (1) & Recent metastatic cancer \\
\hline Older (age >75) (1) & Calculated from birth date \\
\hline Reduced platelet count (1) & Platelets $<75,000$ \\
\hline Re-Bleeding (2) & Prior hospitalization bleeding \\
\hline Hypertension, uncontrolled (1) & $\begin{array}{c}\text { BP not in control; Systolic BP } \geq 160 \\
\text { mmHg }\end{array}$ \\
\hline Anemia (1) & $\begin{aligned} \text { Hematocrit } & <30 \mathrm{~g} / \mathrm{dL} \text { or Hemoglobin } \\
& <10 \mathrm{~g} / \mathrm{dL}\end{aligned}$ \\
\hline Genetic factors (1) & CYP2C $9 * 2$ and/or CYP 2 C $9 * 3$ \\
\hline Elevated risk of fall (1) & $\begin{array}{l}\text { Alzheimer, dementia, Parkinson's, or } \\
\text { schizophrenia }\end{array}$ \\
\hline
\end{tabular}

Stroke (1)

Prior ischemic stroke or brain infraction

AST = aspartate aminotransferase; $\mathrm{ALT}=$ alanine aminotransferase; $\mathrm{Crcl}=$ creatinine clearance; $\mathrm{BP}=$ blood pressure; $\mathrm{CYP}=$ cytochrome $\mathrm{P} 450$ enzyme 


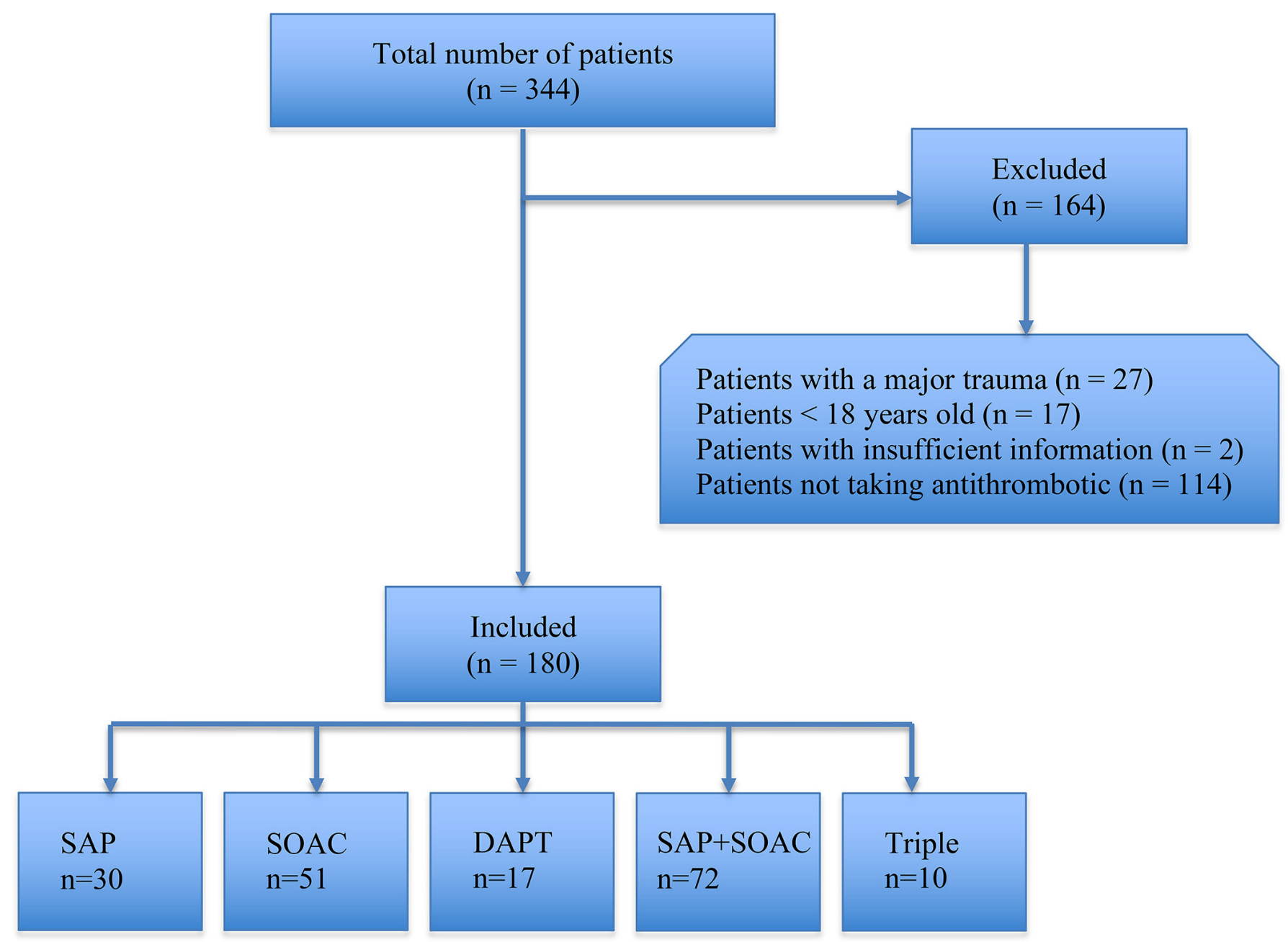

Flow of patients through the study

for patients with ACS and AF who are at high risk of stroke [January 2019]. Appropriate management of these patients still is the source of much debate [Zhao 2011].

The optimal treatment of AF patients with the need for life-long anticoagulation or antiplatelet therapy is evolving. Clinical trials evaluating the safest combinations are lacking, and the appropriate management of those populations is still challenging and a burden to many practitioners [Hansen 2010; Sorensen 2009; Rubboli 2008]. With the introduction of novel oral anticoagulants (NOACs) and new antiplatelet agents, the question regarding the ideal combination will continue to be raised, and the risk of bleeding accompanied with each regimen will be uncertain. Bleeding risk with triple therapies that include NOACs is unknown and a direct comparison of bleeding risk has not been made involving many newer regimens of anticoagulant drug combinations.

Guidelines recommend the assessment of stroke and bleeding risk before initiating anticoagulation in patients with $\mathrm{AF}$ and other cardiovascular diseases [Kearon 2012; Konstantinides 2014]. Multiple scoring systems have been proposed to predict the risk of major bleeding in $\mathrm{AF}$ populations, including the HEMORR ${ }_{2}$ HAGES (Hepatic or Renal
Disease, Ethanol Abuse, Malignancy, Older age, Reduced Platelet Count or Function, Re- Bleeding, Hypertension, Anemia, Genetic Factors, Excessive Fall Risk, and Stroke) [Gage 2006]; HAS-BLED (Hypertension, Abnormal Renal/ Liver Function, Stroke, Bleeding History or Predisposition, Labile International Normalized Ratio, Elderly, Drugs/Alcohol) [Pisters 2010]; and ATRIA (Anticoagulation and Risk factors in Atrial Fibrillation) [Fang 2011]. These act as validated scores in estimating the incidence of relevant bleeding in different patient populations [Apostolakis 2012; Klolk 2016].

The HEMORR ${ }_{2}$ HAGES score consists of eleven criteria, including Hepatic or renal disease, Ethanol abuse, Malignancy, age $>75$ years, Reduced platelet count or function, Rebleeding risk, uncontrolled Hypertension, Anemia, Genetic factors, Excessive fall risk, and Stroke. Each bleeding risk factor weighs 1 point, except for a prior bleed, which weighs 2 points ( $\mathrm{R}$ in the mnemonic). The rate of bleeding increases markedly with the higher score [Gage 2006] (see Table 1).

The HEMORR,HAGES score could be a good indicator for the estimation of bleeding risk in a number of antithrombotic medications, such as NOACs, whereas HAS-BLED might be more directed to warfarin [Gage 2006; Apostolakis 
Table 2. Baseline Characteristics

\begin{tabular}{|c|c|c|c|c|c|c|}
\hline Age, mean (SD) & $69.9(19.4)$ & $62.9(17.6)$ & $69.4(13.9)$ & $71.9(12.9)$ & $78.6(4.8)$ & .0076 \\
\hline Weight $(\mathrm{kg})$, mean (SD) & $72(14)$ & $77(12)$ & $73(20)$ & $80(13)$ & $70(11)$ & .40 \\
\hline Height (cm), mean (SD) & $173(12)$ & $173(10)$ & 171 (14) & $179(14)$ & $177(16)$ & .60 \\
\hline
\end{tabular}

$\mathrm{SAP}=$ single antiplatelet; SOAC = single oral anticoagulant; DAPT = dual antiplatelet; SAP+SOAC = combination of single antiplatelet and single oral anticoagulant; $\mathrm{N}=$ number; $\mathrm{SD}=$ standard deviation

2012; Klolk 2016; Zalesak 2013]. According to our knowledge, no studies have evaluated the HEMORR,HAGES score for patients on DAPT and triple therapy, which makes this study a good start for considering this score in the future with a larger sample size.

Goal of this investigation: We hypothesized that the more antithrombotic regimen potency, the less HEMORR ${ }_{2}$ HAGES score you have. The primary objective of our study is to examine whether a relationship exists between a baseline HEMORR,HAGES score and antithrombotic potency among patients presenting with a bleeding complication by calculating the HEMORR ${ }_{2}$ HAGES score among different regimens.

\section{METHODS}

Study design: This study was a retrospective observational study between November 1, 2013 and August 31, 2015. It was conducted at Banner University Medical Center Tucson (BUMCT) in Tucson, AZ, USA, a 479-bed teaching medical center. The study received institutional review board approval, according to the institution's policy.

Selection of participants: The study enrolled patients admitted to hospital with a diagnosis of bleeding. Patients were included in our study based on an International Classification of Disease, Ninth Revision (ICD-9 code) of $578.0,578.1,578.9,432.1,386.30,386.31,386.39,459.0$, and 853.0 indicating a diagnosis of active bleeding not limited to: intracranial hemorrhage, subdural hematoma, GI bleeding, melena, hemoptysis, hematemesis, retroperitoneal bleeding, or fatal bleeding.

Subjects were eligible in our study if they: (1) were age 18 years or older, and (2) had confirmed active bleeding diagnosis. Subjects were excluded if they: (1) had a major trauma, (2) were not taking antithrombotic medications, and (3) had insufficient information to perform a retrospective review.

The antithrombotic groups in our study included patients who were on the following regimens: single antiplatelet therapy (SAP), single oral anticoagulant therapy (SOAC), dual antiplatelet therapy (DAPT), dual combination (SOAC+SAP), and triple antithrombotic therapy.
In the Sub Group analysis, we ended up combining patients on SAP and SOAC, who were on single antithrombotic therapy, into a new modified Group 1, and combined patients on DAPT and SAP+SOAC, who were on two antithrombotic therapies, into a new modified Group 2 and kept those patients who were on triple therapy.

Data collection - Outcomes: The following information was collected in our study, including but not limited to demographic data, serum creatinine, estimated creatinine clearance using Cockcroft-Gault Equation, name and dose of antithrombotic therapy, appropriateness of antithrombotic medications based on renal dosing for NOACs and INR for warfarin, and total HEMORR ${ }_{2}$ HAGES score. This was based on concomitant disease states, including hepatic or renal disease, ethanol abuse, malignancy, older (age $>75$ years), reduced platelet count or function, re-bleeding risk, hypertension (uncontrolled), anemia, genetic factors (CYP 2C9 single nucleotide polymorphisms), excessive fall risk (including neuropsychiatric disease), and stroke (Table 1). To improve our consistency toward the calculated score, we added one point for each patient who had a history of uncontrolled hypertension defined as systolic $\mathrm{BP} \geq 160 \mathrm{mmHg}$, from at the time of admission to our facility or during any point in the patient's hospitalization.

The primary outcome of this study was to calculate the

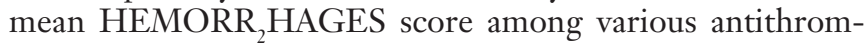
botic regimens.

Data analysis: Categorical variables were evaluated by using a chi-square analysis unless the sample size for a case was less than 5, and in those cases, a Fisher's exact test was used. Normally distributed continuous variables were analyzed by using ANOVA test, and Bonferroni correction test was used for post hock analysis when the difference exists.

\section{RESULTS}

Of the 344 patients identified, 180 patients met the inclusion criteria. Of those, 30 were in the SAP Group, 51 were in the SOAC Group, 17 were in the DAPT Group, 72 were in the dual combination of SAP+SOAC Group, and 10 were in the Triple Therapy Group. Of the 164 patients excluded, 114 were not 
Table 3. Outcomes

\begin{tabular}{|c|c|c|c|c|c|c|}
\hline Hepatic/Renal, N (\%) & $7(23.3 \%)$ & $10(19.6 \%)$ & $3(17.7 \%)$ & $14(19.4 \%)$ & $1(10.0 \%)$ & .93 \\
\hline Malignancy, N (\%) & $8(26.7 \%)$ & $12(23.5 \%)$ & $3(17.7 \%)$ & $10(13.9 \%)$ & $0(0.0 \%)$ & .24 \\
\hline Age $>75$ years, $N(\%)$ & $13(43.3 \%)$ & $10(19.6 \%)$ & $7(41.2 \%)$ & $36(50.0 \%)$ & $7(70.0 \%)$ & .003 \\
\hline Re-bleeding, N (\%) & $7(23.3 \%)$ & $18(35.3 \%)$ & $3(17.7 \%)$ & $11(15.3 \%)$ & $3(30.0 \%)$ & .12 \\
\hline Hypertension, N (\%) & $24(80.0 \%)$ & $31(60.8 \%)$ & $16(94.1 \%)$ & $67(93.1 \%)$ & $10(100 \%)$ & $<.001$ \\
\hline Anemia, N (\%) & $17(56.7 \%)$ & $31(60.8 \%)$ & $7(41.2 \%)$ & $43(59.7 \%)$ & $2(20.0 \%)$ & .11 \\
\hline Fall, N (\%) & $3(10.0 \%)$ & $2(3.9 \%)$ & $0(0.0 \%)$ & $1(1.4 \%)$ & $0(0.0 \%)$ & .20 \\
\hline Stroke, N (\%) & $7(23.3 \%)$ & $4(7.8 \%)$ & $3(17.7 \%)$ & $11(15.3 \%)$ & $1(10.0 \%)$ & .39 \\
\hline
\end{tabular}

$\mathrm{SAP}=$ single antiplatelet; SOAC = single oral anticoagulant; DAPT $=$ dual antiplatelet; $\mathrm{SAP}+\mathrm{SOAC}=$ combination of single antiplatelet and single oral anticoagulant; $\mathrm{N}$ = number; $\mathrm{SD}=$ standard deviation

on antithrombotic therapy, 27 had trauma, 17 were less than 18 years old, and 5 had insufficient information (Figure).

Overall, patients on SOAC therapy significantly were younger than the other groups. The mean age $( \pm \mathrm{SD})$ was $(62.9$ \pm 16.7 years; $P=.0076$ ) (Table 2). Patients with a history of hypertension significantly were less represented in the SOAC Group compared with the other groups $(60.8 \% ; P \leq .0001)$ (Table 3). The most common antiplatelet used in this study was aspirin $(68.9 \%)$, followed by clopidogrel (14.4\%) and ticagrelor $(3.3 \%)$. We did not have any patients taking prasugrel in our study. The most common anticoagulant used was warfarin $(39.9 \%)$, followed by rivaroxaban $(23.9 \%)$, followed by dabigatran $(7.8 \%)$, and apixaban $(2.8 \%)$. We had only one patient on edoxaban $(0.6 \%)$. The most frequently used combination for DAPT was aspirin and clopidogrel (82.4\%). The most common triple antithrombotic therapy was aspirin, clopidogrel, and dabigatran (30\%). Finally, we found that $(83.9 \%)$ of our subjects received appropriate dosing of the antithrombotic therapy, including therapeutic INR at the time of bleeding.

There was no significant difference among the five groups in the following disease states: history of hepatic or renal disease, ethanol abuse, history of rebleeding, history of fall, and history of stroke. We found a significant difference between the SAP and SOAC groups in the history of reduced platelet count or function $6.7 \%$ versus $19.6 \% ; P=.0003$. Also, there was a significant difference in patients older than 75 years between the SOAC and dual combination SAP+SOAC groups $19.6 \%$ versus $41.2 \%$ and between the SOAC and Triple Therapy groups $19.6 \%$ versus $70 \% ; P=.003$. In addition, patients with a history of hypertension significantly were less represented in the SOAC Group compared with the other groups; $P \leq .0001$ (see Table 3).

\section{Main Results}

The HEMORR 2 HAGES score was highest in the SAP Group compared with the other groups $(3.23 \pm 1.1)$.
Interestingly, patients in the DAPT Group had the lowest HEMORR ${ }_{2}$ HAGES score compared with the other groups $(2.59 \pm 1.2)$. However, there was no significant difference among all the groups in the HEMORR,HAGES score $(P=.36)$ (see Table 3$)$.

In the Sub Group analysis, we found that the HEM$\mathrm{ORR}_{2}$ HAGES score was highest in the modified Group 1 compared with the modified Group 2 and Triple Group, $3.06 \pm 1.1$ versus $2.80 \pm 1.1$ versus $2.70 \pm 1.6$, respectively; $P=.29$. We found a trend of decreasing the mean HEMOR$\mathrm{R}_{2}$ HAGES score as we added more antithrombotic therapy; however, there still was no statistical difference among the three groups (see Table 4).

\section{DISCUSSION}

The ideal combination of treatments in patients with atrial fibrillation and coronary artery disease remains unclear due to lack of a head-to-head trail among the new agents; therefore, it is important to understand the associated outcomes of these various agents [Hansen 2010; Sorensen 2009; Rubboli 2008; Kearon 2012; Konstantinides 2014]. Since the major concern with initiating all antithrombotic agents is the high risk of bleeding, the availability of various bleeding risk score can be crucial in determining whether the benefits outweigh the risks.

HEMORR,HAGES bleeding score originally was developed from the results of the National Registry of Atrial Fibrillation (NRAF) to quantify the risk of bleeding in elderly patients with atrial fibrillation. In its study, the mean HEMORR,HAGES score was 1.9 for warfarin group, 3.1 for aspirin group $(P<.001)$ [Gage 2006]. The results of NRAF highlighted the validity of using HEMORR_HAGES score as a good predictor of bleeding in patients who were prescribed warfarin or aspirin, but it 
Table 4. Sub Group Analysis

\begin{tabular}{lccc}
\hline Variable & Modified Group 1 SAP+SOAC N =81 & Modified Group 2 DAPT + (SAP+SOAC) N=89 & Group 3 Triple N = 10 \\
\hline HEMORR ${ }_{2}$ HAGES score, mean (SD) & $3.06(1.1)$ & $2.80(1.1)$ & $2.70(1.6)$ \\
\hline
\end{tabular}

$\mathrm{SAP}=$ single antiplatelet; $\mathrm{SOAC}=$ single oral anticoagulant; $\mathrm{DAPT}=$ dual antiplatelet; $\mathrm{SAP}+\mathrm{SOAC}=$ combination of single antiplatelet and single oral anticoagulant; $\mathrm{N}$ = number; $\mathrm{SD}=$ standard deviation

may be a valid score in patients prescribed newer anticoagulants as well [Gage 2006; Zalesak 2013].

According to our knowledge, there only was one study

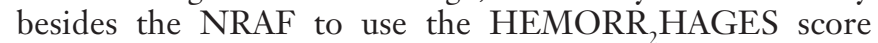
to examine persistence rate in newly diagnosed nonvalvular atrial fibrillation patients treated with warfarin versus dabigatran as their initial oral anticoagulation. The mean HEMORR ${ }_{2}$ HAGES score in this study was $2.4 \pm 1.5$ in warfarin group versus $2.7 \pm 1.6$ in dabigatran group; $P<.001$ [Zalesak 2013].

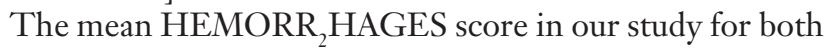
the SAP and SOAC groups was higher than what was reported in the two studies mentioned above [Gage 2006; Zalesak 2013]. One explanation is the inconsistency in reporting the different variables in the score, which may have affected some of the results. For example, history of uncontrolled hypertension (systolic BP $\geq 160 \mathrm{mmHg}$ ) could be considered at only the time of admission to our facility or at any point during patient's hospitalization. We ended up adding one point for any patient, of which $148(82.2 \%)$, had any reading of systolic $\mathrm{BP} \geq 160 \mathrm{mmHg}$ during their entire admission to improve our consistency. At this point so far, we could not find any

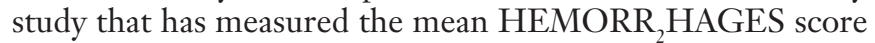
for patients on dual or triple antithrombotic therapy; therefore, we could not estimate the sample size for each group.

It is unclear why patients in the DAPT Group had the lowest HEMORR HAGES score in our study. The best explanation would be those 17 patients in the group were sicker than the other groups, though there were limited subjects in this group. We hypothesized that subjects in the Triple Therapy Group would have the lowest score since their risk of bleeding could be higher compared with one or two antithrombotic agents [Orford 2004; Andrade 2013; Hansen 2010; Sorensen 2009; Rubboli 2008]. This hypothesis was true in the Sub Group analysis, when we compared single versus dual versus triple therapy as Triple Therapy Group had the lowest score.

Individual elements of HEMORR $\mathrm{HAGES}_{2}$ score to predict bleeding showed difference in patients on SOAC therapy. This might be explained because the median age in the SOAC Group significantly was lower than the dual combination SOAC+SAP and Triple Therapy groups. In addition, patients with a history of hypertension and age $>75$ proportionately were less represented in the SOAC Group compared with other groups.

It should be noted that our study included patients with various thromboembolic diseases and did not only focus on atrial fibrillation patients. This concept allowed us to test the

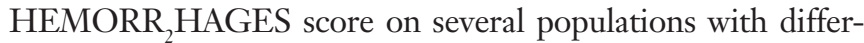
ent options of treatment. Thus, it helped us examine the function of this bleeding score with complicated regimens. However, there was no difference among the different groups mainly due to the low number of subjects in this study. The availability of a well-designed study with a larger population could be a promising idea for further testing of this bleeding score.

Several limitations should be considered in this study. First, we can't generalize the results of this study to all patients with active bleeding because we conducted this study in a single center. Second, the use of ICD-9 codes for the diagnosis of active bleeding in our institution might not capture all eligible patients. Third, we had a limited number of subjects in some of the antithrombotic regimens, which could affect the study power of our results. In addition, we could not find any prior study that had a controlled mean HEMORR 2 HAGES score for different regimens as we used in our study. Finally, genetic risk factor for bleeding was not readily available in our study because this test was not available in our institution at the time that this study was conducted.

In conclusion, HEMORR 2 HAGES score did not differentiate antithrombotic potency amongst groups with various regimens. This study emphasized that the decision to initiate antithrombotic therapy on patients at high risk of bleeding should always considered the harms and benefits.

\section{REFERENCES}

Andrade JG, Deyell MW, Khoo C, et al. 2013. Risk of bleeding on triple antithrombotic therapy after percutaneous coronary intervention/stenting: a systematic review and meta-analysis. Can J Cardiol 29:204e212.

Apostolakis S, Lane DA, Guo Y, et al. 2012. Performance of the HEM$\mathrm{ORR}_{2}$ HAGES, ATRIA, and HAS-BLED bleeding risk-prediction scores in patients with atrial fibrillation undergoing anticoagulation: the AMADEUS (evaluating the use of SR34006 compared to warfarin or acenocoumarol in patients with atrial fibrillation) study. J Am Coll Cardiol 60:861-7.

D’Ascenzo F, Bollati M, Clementi F, et al. 2013. Incidence and predictors of coronary stent thrombosis: evidence from an international collaborative meta-analysis including 30 studies, 221,066 patients, and 4276 thromboses. Int J Cardiol 167:575e584.

Fang MC, Go AS, Chang Y, et al. 2011. A new risk scheme to predict warfarin-associated hemorrhage: the ATRIA (Anticoagulation and Risk Factors in Atrial Fibrillation) study. J Am Coll Cardiol 58: 395- 401.

Gage BF, Yan Y, Milligan PE, et al. 2006. Clinical classification for predicting hemorrhages: results from the National Registry of Atrial Fibrillation (NRAF). Am Heart J. 151:713-9. 
Hansen ML, Sorensen R, Clausen MT, et al. 2010. Risk of bleeding with single, dual, or triple therapy with warfarin, aspirin, and clopidogrel in patients with atrial fibrillation. Arch Intern Med 170:1433.

January CT, Wann LS, Calkins H, et al. 2019. AHA/ACC/HRS focused update of the 2014 AHA/ACC/HRS guideline for the management of patients with atrial fibrillation: A report of the American college of cardiology/American heart association task force on clinical practice guidelines and the heart rhythm society. Heart Rhythm Jan 28. pii: S1547-5271(19)30037-2.

Kearon C, Akl EA, Comerota AJ, Prandoni P, Bounameaux H, Goldhaber SZ, Nelson ME, Wells PS, Gould MK, Dentali F, Crowther M, Kahn SR. 2012. Antithrombotic therapy for VTE disease: antithrombotic therapy and prevention of thrombosis, 9th ed: American College of Chest Physicians evidence-based clinical practice guidelines. Chest 141:e419S-e494S.

Klolk FA, Niemann C, Dellas C, et al. 2016. Performance of five different bleeding-prediction scores in patients with acute pulmonary embolism. J Thromb Thrombolysis 2:312-20.

Konstantinides SV, Torbicki A, Agnelli G, Danchin N, Fitz- Maurice D, Galie N, Gibbs JS, Huisman MV, Humbert M, Kucher N, Lang I, Lankeit M, Lekakis J, Maack C, Mayer E, Meneveau N, Perrier A, Pruszczyk P, Rasmussen LH, Schindler TH, Svitil P, Vonk NA, Zamorano JL, Zompatori M. 2014. 2014 ESC guidelines on the diagnosis and management of acute pulmonary embolism: the task force for the diagnosis and management of acute pulmonary embolism of the European Society of Cardiology (ESC) Endorsed by the European Respiratory Society (ERS). Eur Heart J 35:3033-3069.
Lamberts M, Olesen JB, Ruwald MH, et al. 2012. Bleeding after initiation of multiple antithrombotic drugs, including triple therapy, in atrial fibrillation patients following myocardial infarction and coronary interventions: a nationwide cohort study. Circulation 126:1185-1193.

Orford JL, Fasseas P, Melby S, et al. 2004. Safety and efficacy of aspirin, clopidogrel, and warfarin after coronary stent placement in patients with an indication for anti- coagulation. Am Heart J 147:463e467.

Pisters R, Lane DA, Nieuwlaat R, et al. 2010. A novel user-friendly score (HAS-BLED) to assess 1-year risk of major bleeding in patients with atrial fibrillation: the Euro Heart Survey. Chest 138:1093-100.

Rubboli A, Halperin JL, Airaksinen KE, et al. 2008. Antithrombotic therapy in patients treated with oral anticoagulation undergoing coronary artery stenting. An expexrt consensus document with focus on atrial fibrillation. Ann Med 40:428.

Sorensen R, Hansen ML, Abildstrom SZ, et al. 2009. Risk of bleeding in patients with acute myocardial infarction treated with different combinations of aspirin, clopidogrel, and vitamin $\mathrm{K}$ antagonists in Denmark: a retrospective analysis of nationwide registry data. Lancet 374:1967.

Zalesak M, Siu K, Francis K, et al. 2013. Higher persistence in newly diagnosed nonvalvular atrial fibrillation patients treated with dabigatran versus warfarin. Circ Cardiovasc Qual Outcomes 5:567-74.

Zhao HJ, Zheng ZT, Wang ZH, et al. 2011. "Triple therapy" rather than "triple threat": a meta-analysis of the two antithrombotic regimens after stent implantation in patients receiving long-term oral anticoagulant treatment. Chest 139:260e270. 